\title{
To Study and Determine the Role of Anterior Segment Optical Coherence Tomography and Ultrasound Biomicroscopy in Corneal and Conjunctival Tumors
}

\author{
Katleen Janssens, ${ }^{1}$ Michelle Mertens, ${ }^{1}$ Noémie Lauwers, ${ }^{2}$ Rob J. W. de Keizer, ${ }^{2,3}$ \\ Danny G. P. Mathysen, ${ }^{1,2}$ and Veva De Groot ${ }^{1,2}$ \\ ${ }^{1}$ University of Antwerp, Antwerp, Belgium \\ ${ }^{2}$ Department of Ophthalmology, University Hospital Antwerp, Edegem, Belgium \\ ${ }^{3}$ Department of Ophthalmology, LUMC, Leiden, Netherlands
}

Correspondence should be addressed to Michelle Mertens; michelle.mertens@student.uantwerpen.be

Received 17 June 2016; Accepted 23 October 2016

Academic Editor: Karim Mohamed-Noriega

Copyright (C) 2016 Katleen Janssens et al. This is an open access article distributed under the Creative Commons Attribution License, which permits unrestricted use, distribution, and reproduction in any medium, provided the original work is properly cited.

\begin{abstract}
Purpose. To analyze and describe corneal and conjunctival tumor thickness and internal characteristics and extension in depth and size and shape measured by two noninvasive techniques, anterior segment optical coherence tomography (AS-OCT) and ultrasound biomicroscopy (UBM). Design. Systematic review. Methods. This systematic review is based on a comprehensive search of 4 databases (Medline, Embase, Web of Science, and Cochrane Library). Articles published between January 1, 1999, and December 31, 2015, were included. We searched for articles using the following search terms in various combinations: "optical coherence tomography", "ultrasound biomicroscopy", "corneal neoplasm", "conjunctival neoplasm", "eye", "tumor" and "anterior segment tumors". Inclusion criteria were as follows: UBM and/or AS-OCT was used; the study included corneal or conjunctival tumors; and the article was published in English, French, Dutch, or German. Results. There were 14 sources selected. Discussion. Several studies on the quality of AS-OCT and UBM show that these imaging techniques provide useful information about the internal features, extension, size, and shape of tumors. Yet there is no enough evidence on the advantages and disadvantages of UBM and AS-OCT in certain tumor types. Conclusion. More comparative studies are needed to investigate which imaging technique is most suitable for a certain tumor type.
\end{abstract}

\section{Introduction}

Since the early nineties ultrasound biomicroscopy (UBM) has been used for the imaging of the anterior eye segment and was soon discovered to be useful in the evaluation of superficial tumors. Anterior segment optical coherence tomography (AS-OCT) became available in the 21st century. One of the first series on the use of OCT in the evaluation of conjunctival tumors was published by Buchwald et al., in 2003 [1], followed by De Keizer and Razzaq in 2007 [2].

Corneal and conjunctival tumors can be visualized by AS-OCT and UBM, two noninvasive imaging techniques. Hereby, tumor thickness and internal characteristics and extension in depth and size and shape can be measured. Several small series have been published, evaluating the use and the quality of AS-OCT or UBM in assessing these tumors. It is still not clear which technique to use in certain tumor types. We therefore conducted a literature search in order to find an answer to the following question: how accurate are AS-OCT and UBM in determining tumor margins and tumor depth of conjunctival and corneal tumors and can they provide additional information guiding the diagnosis?

\section{Material and Methods}

2.1. Anterior Segment Optical Coherence Tomography. ASOCT is an examination technique that uses reflected light waves in order to reconstruct a cross section of the examined tissue. Time-domain OCT measures the electromagnetic radiation in function of the time. This investigation can 
make up to 400 axial scans per second and has an axial resolution of $8-10 \mu \mathrm{m}$. Spectral-domain OCT measures the wavelength of the reflected light and compares the image with a reference point by means of mirrors, which allows it to measure faster. This imaging technique also measures electromagnetic radiation and can make up to 25.000-50.000 scans per second. It has an axial resolution of 5-7 $\mu \mathrm{m}$. In comparison, spectral-domain OCT obtains more data in less time and with higher axial resolution $[3,4]$. OCT of the anterior segment can achieve a $9-10 \mu \mathrm{m}$ axial and $15 \mu \mathrm{m}$ transverse resolution [5]. This enables it to visualize smaller details compared to those shown on ultrasound or MRI [6, 7]. Ultrahigh Resolution OCT (UHR-OCT) uses a similar technique to OCT but results in a higher resolution $(3 \mu \mathrm{m}$ axial resolution) [8-10].

Advantages and Disadvantages. OCT has many important advantages. It is a widely available noncontact method without ionizing radiation. As a result, there are no risk of eye damage and no discomfort for the patient. OCT directly renders high resolution images while the examination takes less than 5 minutes and can be performed by an optometrist. In comparison with other competing imaging techniques, AS-OCT has a low penetration depth of 1-3 mm but offers a high axial resolution due to the use of short wavelength light ( $\pm 830 \mathrm{~nm}$ mostly) $[3,5,11]$. AS-OCT is ideal for imaging structures from the surface of the eye to the level of the iris [5]. A disadvantage of AS-OCT is that it cannot visualize structures behind pigmented lesions, like the iris, and cannot visualize early pathological changes smaller than $5 \mu \mathrm{m}$ such as early dysplasia $[5,7]$.

2.2. Ultrasound Biomicroscopy. UBM is an examination technique that uses sound waves to analyze the structures as in the classic ultrasound investigation. However, in UBM a higher frequency is used which consequently allows more detail but less penetration into the tissue [12]. For the anterior segment, transducers are used with a frequency of 35-50 MHz. UBM has an axial resolution of $42 \mu \mathrm{m}$ and a tissue penetration of 4-5 mm [5, 13].

Advantages and Disadvantages. The most important advantage of UBM is that it can penetrate opaque tissue [14]. In contrast, AS-OCT is unable to do this. UBM also has a broad field of view [15]. UBM is ideal for imaging structures from the surface of the eye to the anterior vitreous [5]. Disadvantages are the limited penetration depth of 4-5 mm and the limited depth resolution. UBM requires topical anesthesia and the application of an eyecup filled with fluid in contact with the eye surface causing mild discomfort for the patient. Unfortunately the accessibility to UBM is limited to the larger centers.

2.3. Methods. We performed a specific literature search of peer reviewed published journal articles in the following stages.

Stage 1. Comprehensive search of 4 databases (Medline, Embase, Web of Science, and Cochrane Library). Articles published between January 1, 1999, and December 31, 2015, were included. We searched for articles using the following search terms in various combinations: "optical coherence tomography", "ultrasound biomicroscopy", "corneal neoplasm", "conjunctival neoplasm", "eye", "tumor", and "anterior segment tumors".

Stage 2. Articles were first selected based on title and subsequently on abstract and full text. Inclusion criteria were as follows: UBM and/or AS-OCT was used; the study included corneal or conjunctival tumors; the article was published in English, French, Dutch, or German. We could include 14 articles.

The flow diagram of the literature search is shown in Figure 1.

\section{Results}

We analyzed 14 papers: 6 studies on UBM, 6 on OCT, and 2 studies that compared the two imaging techniques.

All studies are listed in Tables 1, 2, and 3.

3.1. UBM. Lanzl et al. [16], Grant and Azar [17], and Hoops et al. [18] all studied the use of UBM in limbal dermoids, respectively, on 2,1 , and 8 patients. They all found a hyperreflective lesion compared to the cornea. They concluded that UBM is a useful preoperative tool in limbal dermoids [16, 17] but insufficient in some cases to measure corneal penetration [17].

Buchwald et al. demonstrated in 28 patients with solid tumors of the conjunctiva that UBM can be an additional diagnostic tool, for example, to determine the margins of the solid tumors or cysts [19].

Similarly to the study of Buchwald et al., Lin et al. proved in 2 cases that UBM is a useful tool to show cysts in conjunctival lesions. This technique could be used for delineating the extent of the lesion before excision [20].

Ho et al. studied the assessment of tumor thickness in three conjunctival melanomas by means of UBM. They concluded that high frequency UBM is useful for estimating tumor thickness in conjunctival melanomas and can be used to determine the tumor depth prior to surgical resection [21].

3.2. AS-OCT. The study of Shields et al. on 22 conjunctival nevi demonstrated that all margins of conjunctival nevi, including the deep borders, could be visualized by AS-OCT. AS-OCT images showed a high resolution in $100 \%$ of anterior borders and $82 \%$ of posterior borders [22]. Some of the images were affected by deep optical shadowing, especially in pigmented nevi (86\%). The sensitivity of AS-OCT for the detection of intrinsic cysts in a conjunctival nevus is $80 \%$, the specificity is $100 \%$, the positive predictive value is $100 \%$, and the negative predictive value is $60 \%$. Thus, AS-OCT ensures high resolution images of conjunctival nevi, it can delineate the borders of the lesion, and it can demonstrate the presence of intralesional cysts [22]. The main drawback of OCT is the presence of optical shadowing in pigmented nevi. The thickness of nevi in the study ranged from $0.1 \mathrm{~mm}$ to $1.7 \mathrm{~mm}$ measured with AS-OCT [22]. 

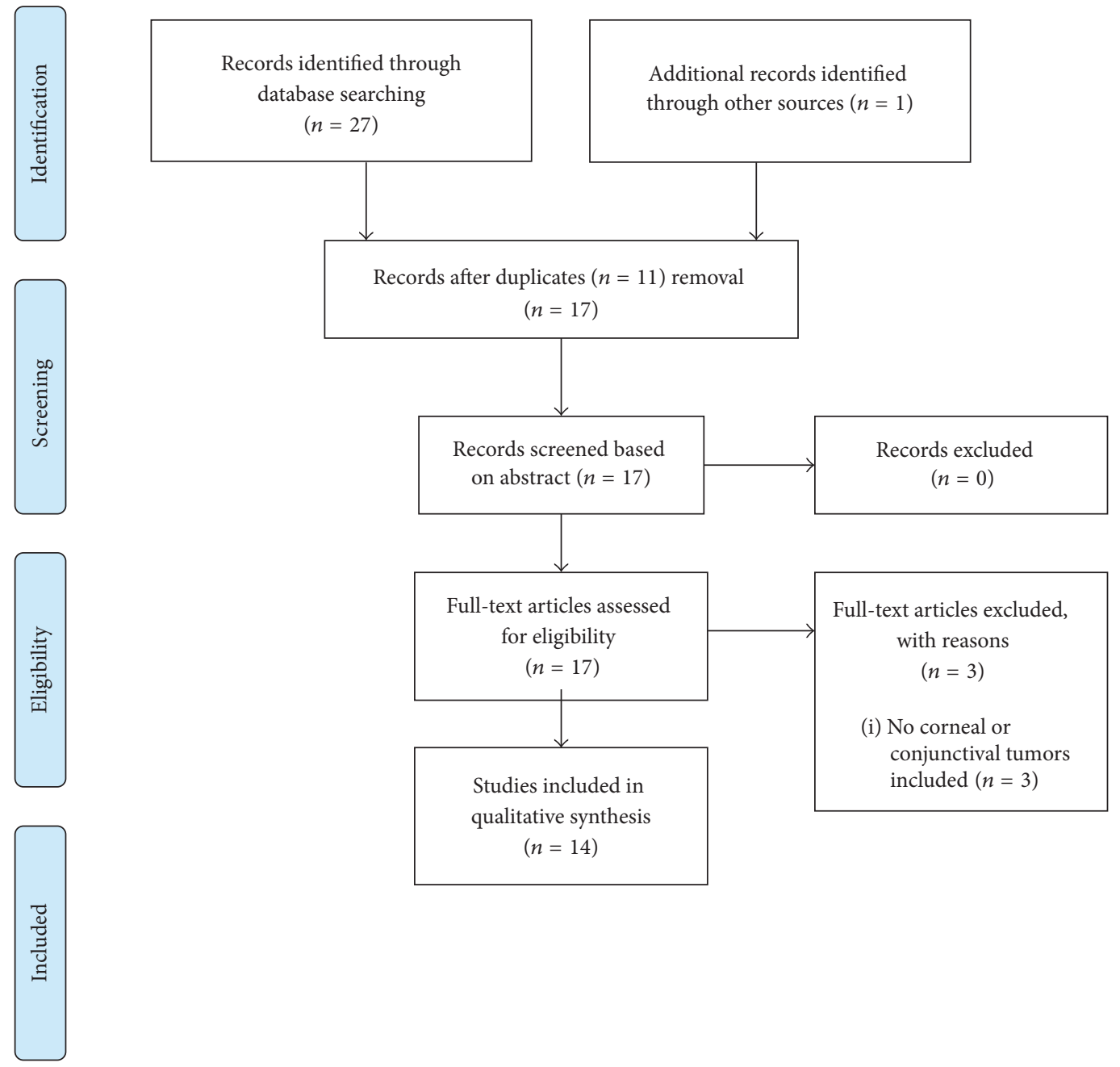

FIGURE 1: Flow diagram of literature search.

Welch et al. studied the difference between the measurements of a pterygium by slit-lamp examination and by ASOCT imaging [23]. When measuring the distance from the apex of the pterygium to the limbus in 13 eyes, AS-OCT gave significantly better reproducible results. Therefore, they concluded that AS-OCT allows us to accurately determine the extension of a pterygium on the cornea [23].

Shousha et al. studied the use of UHR-OCT in the diagnosis and follow-up of conjunctival and corneal intraepithelial neoplasia (CCIN). UHR-OCT images of the 7 lesions discerned a thickened hyperreflective epithelium and abrupt transition from normal to hyperreflective epithelium. Their results demonstrated that macroscopically resolved residual tumor nodules can be visualized by UHR-OCT. They concluded that UHR-OCT is useful for guiding diagnosis and treatment follow-up of CCIN [9].

The results of the study of Kieval et al. showed that UHR-OCT of the anterior segment could be an accurate tool in differentiating ocular squamous cell carcinoma from pterygium [8]. The difference in measured epithelial thickness allows ophthalmologists to make a distinction. The average epithelial thickness in the 17 epithelial squamous cell carcinomas (SCC) was $346 \mu \mathrm{m}$, compared to $101 \mu \mathrm{m}$ in the 17 pterygia. Using a cut-off value of $142 \mu \mathrm{m}$ results in a sensitivity of $94 \%$ and a specificity of $100 \%$ [8] in differentiating SCC from pterygia.

In another study of Shousha et al., the use of UHROCT in the diagnosis of 54 ocular surface lesions was studied. It was demonstrated that when the clinical diagnosis of ocular surface lesions was uncertain, UHR-OCT images provided optical signs indicating more specific diagnosis and management. They concluded that this imaging technique can visualize the structure and location of the lesion and as such can aid in guiding the diagnosis and management [10].

Nanji et al. studied the use of high resolution, spectraldomain optical coherence tomography (HR-OCT) in the diagnosis of corneal and conjunctival pathologies, with a focus on malignant lesions. In this pilot study on 82 lesions, they concluded that HR-OCT was helpful to determine the etiology and to differentiate between multiple ocular surface lesions, including ocular surface squamous neoplasia, pterygium, nevi, and melanoma, as well as to evaluate resolution 


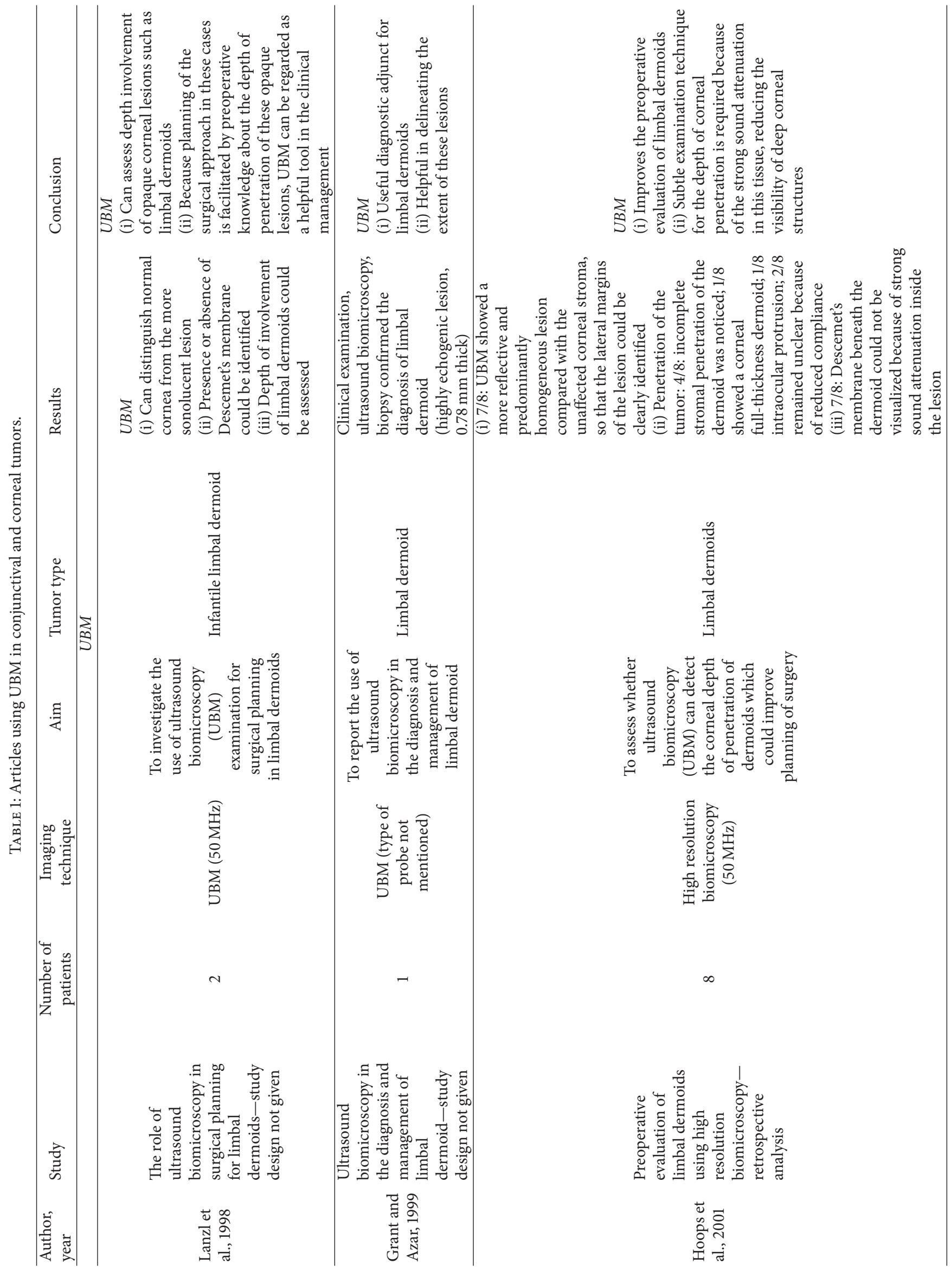




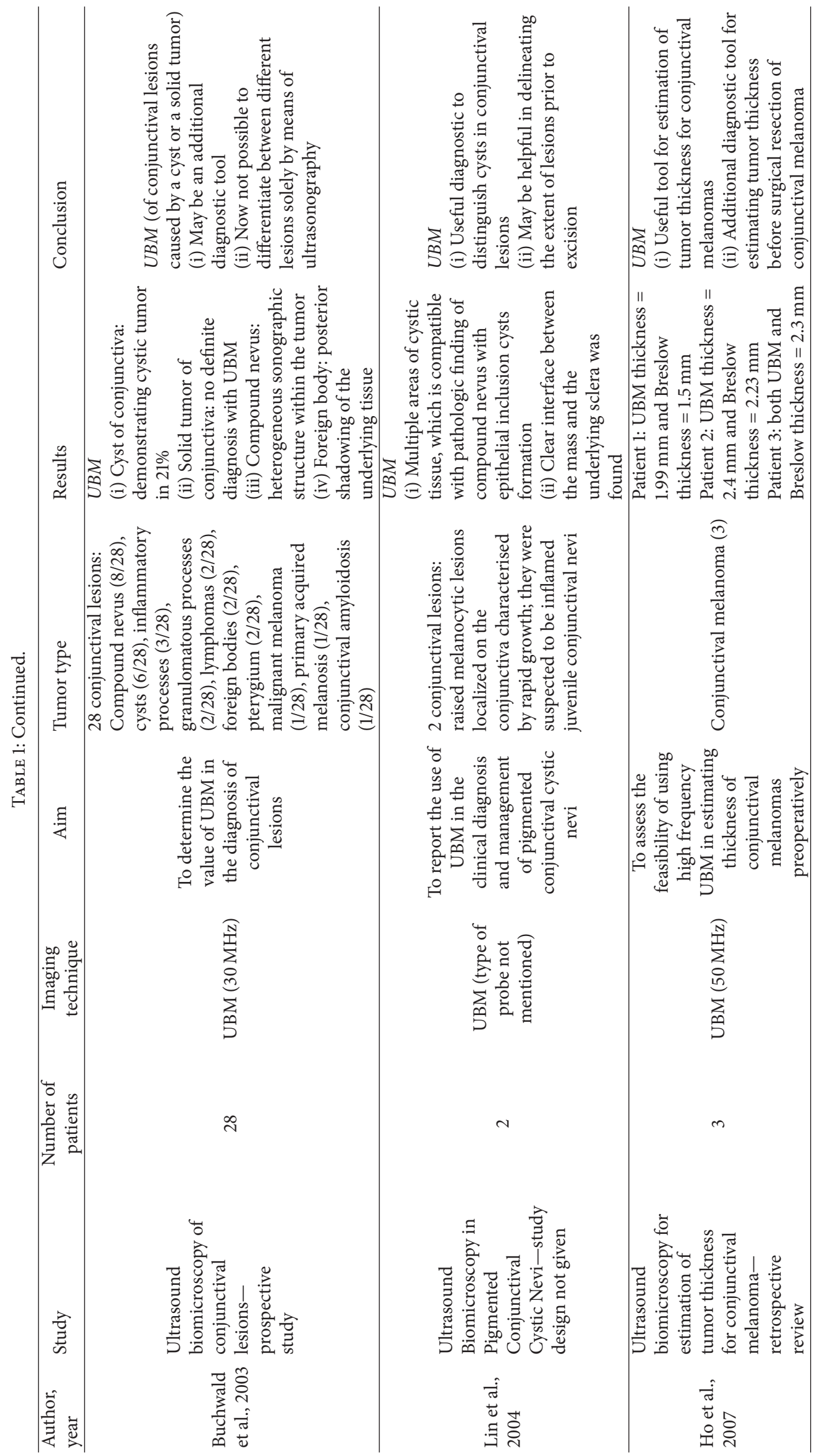




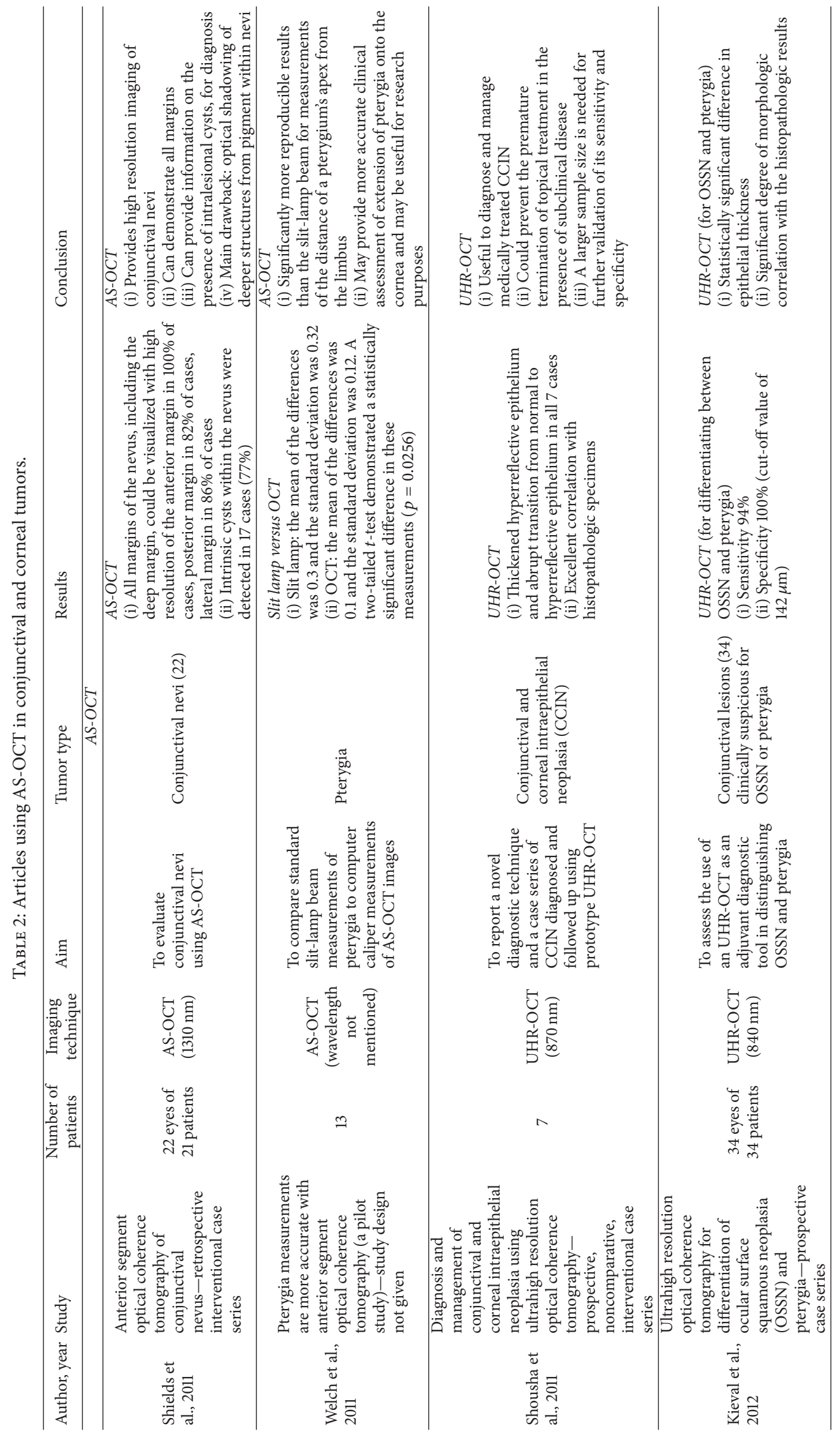




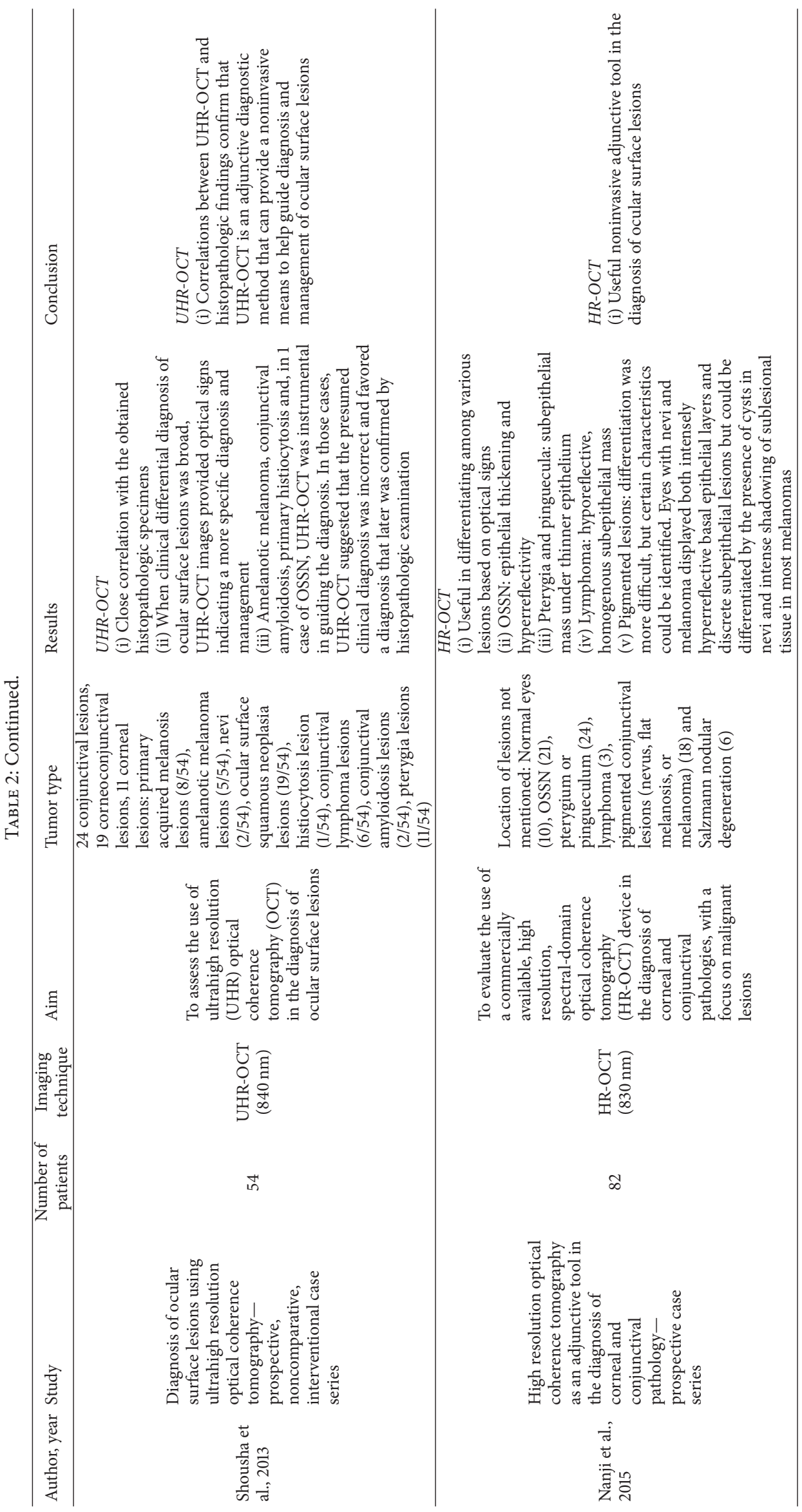




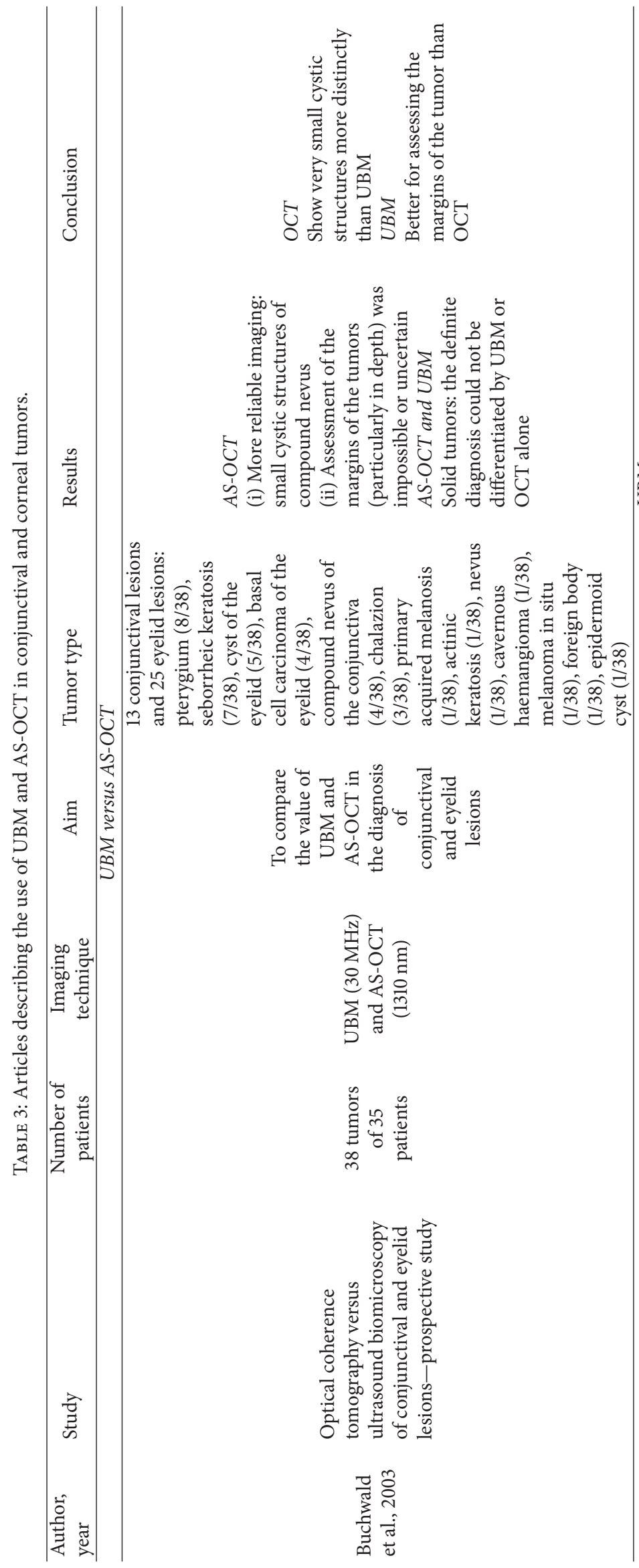

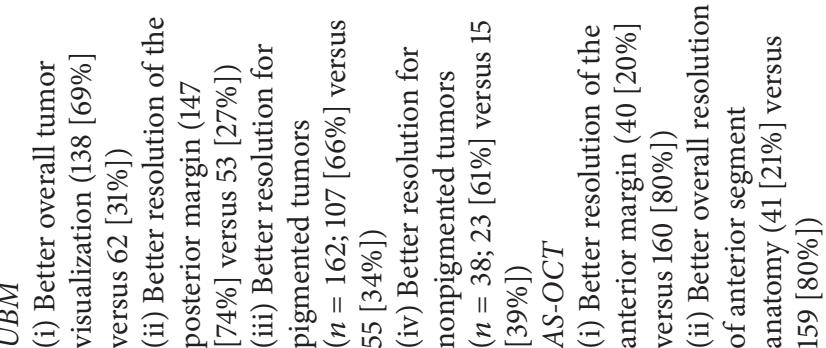

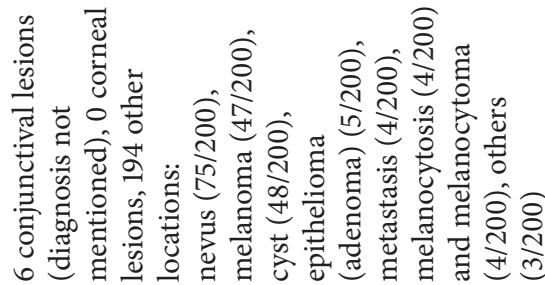

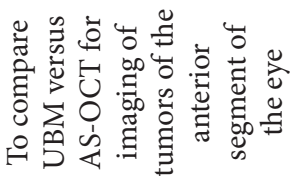

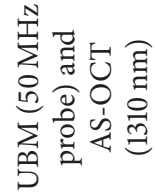

¿

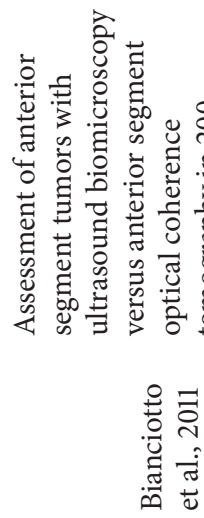


after treatment. However, this imaging technique was less useful in evaluating pigmented lesions. Even though this imaging technique cannot replace either clinical evaluation or histopathologic diagnosis, it can be an important aid in determining the diagnosis of ocular surface pathology and in determining disease resolution [24].

3.3. UBM and AS-OCT. Buchwald et al. studied 13 conjunctival and 25 eyelid lesions. The authors concluded that, in solid tumors, the final diagnosis cannot be made based on UBM or AS-OCT alone [1]. In general, when using ASOCT it was impossible or uncertain to determine the tumor depth. When comparing AS-OCT and UBM, AS-OCT is the better imaging technique for small cystic structures. This is especially useful for nevi as they often contain small cysts. In contrast, UBM is a better technique to determine the tumor margins [1].

Bianciotto et al. studied 200 eyes with anterior segment tumors. Even though they only included 6 conjunctival tumors, their study resulted in interesting conclusions. Comparison of UBM and AS-OCT showed that UBM had a better tumor visualization and better resolution of the posterior margin. UBM also had a better resolution for pigmented as well as for nonpigmented tumors. However, AS-OCT had better resolution of the anterior border and better resolution of the anterior segment anatomy. Posterior tumor shadowing was rarely found in UBM images and more common in ASOCT. The image quality was good in UBM but less in ASOCT. This study shows that AS-OCT is superior to UBM for the imaging of conjunctival lesions, because AS-OCT offers a higher resolution and conjunctival lesions are superficial and mostly not pigmented. Their findings demonstrate that AS-OCT is affected by optical shadowing in large pigmented lesions. AS-OCT uses light, which is more comfortable for the patient, but the light is blocked by opaque tissues which results in lower penetration depth. This is a crucial factor in the evaluation and treatment of anterior segment tumors. They conclude that AS-OCT is a useful tool in the evaluation of superficial nonpigmented lesions of the eye, although ASOCT suffers from poor resolution and shadowing in large or pigmented lesions. In comparison, UBM can penetrate better through the lesion, which results in better visualization of the posterior tumor border and whole tumor configuration [25].

\section{Discussion}

These studies showed that AS-OCT and UBM both have their advantages and disadvantages because of their specific characteristics. None can replace histopathological examination for diagnosis but they both give useful information helping in the differential diagnosis $[1,19]$. Both noninvasive imaging techniques provide useful information about the thickness and internal characteristics and extension in depth and size and shape of conjunctival and corneal tumors.

Although AS-OCT technically has a higher resolution than UBM $[9,10]$, UBM seems to be superior in accuracy of tumor visualization, quality of the image, resolution of the posterior margin, and resolution of pigmented and nonpigmented tumors $[1,24,25]$. UBM is also able to visualize all margins more often than AS-OCT $[1,25]$. Therefore UBM is a useful tool in delineating the margins and measuring the extent of a conjunctival lesion before surgical excision [20]. Another advantage of UBM is that tumor shadowing is rarely seen, while this is much more frequently seen on AS-OCT $[8,9,24,25]$. For this reason, AS-OCT is not preferable for the visualization of large pigmented lesions [25]. In contrast, AS-OCT provides a better resolution of the anterior margin and anterior segment anatomy [25].

For thicker lesions as a limbal dermoid, UBM is the preferred technique. UBM has proven to be valuable in measuring the depth and extension of dermoids $[17,18]$ and also in establishing a differential diagnosis [17]. Since UBM can accurately measure depth of invasion, it is therefore very useful in the preoperative evaluation of a limbal dermoid $[16,18]$.

Conjunctival nevi often contain typical small cysts. Lin et al. demonstrated that UBM is useful in visualizing these cysts in pigmented conjunctival lesions [20]. Shields et al. and Buchwald et al. found that AS-OCT can identify these small cystic structures more accurately than UBM, and, therefore, AS-OCT is a useful tool in investigating these lesions $[1,22]$. AS-OCT also has a high correlation with clinical examination and histopathology in visualizing these intrinsic cysts [22]. It was also found that AS-OCT can visualize all margins of conjunctival nevi, even the deep margins, although deep optical shadowing is often seen, especially in pigmented nevi [22]. For conjunctival nevi we can conclude that AS-OCT seems to be more accurate in assessing the extent of these tumors as long as the nevus is not very thick and not heavily pigmented.

Ho et al. investigated conjunctival melanomas and found that UBM is a very good technique for determining the posterior margin and estimating tumor thickness. Therefore, UBM is useful in determining the excision depth important in the planning of a surgical resection [21]. AS-OCT was not compared to UBM in conjunctival melanomas.

Concerning squamous cell carcinoma (SCC), HR-OCT was found to be useful in the differentiation between SCC and similar lesions like amelanotic melanoma and corneal fibrosis $[10,24]$. Studies which concentrated on the differentiation between SCC and pterygia concluded that AS-OCT is a good diagnostic tool to differentiate SCC from pterygia $[8,10,24]$. AS-OCT can differentiate between these two lesions by measuring the difference in epithelial thickness [8]. Furthermore, the authors found that, for SCC as well as pterygia, there is a good correlation between the findings on UHR-OCT and histopathology [8]. Also Nanji et al. found that for SCC HR-OCT correlated well with histopathology [24]. HR-OCT can also be used for the monitoring of the resolution of SCC during therapy. In this way, HR-OCT can detect subtle residual epithelial thickening which is not visible on clinical examination. This prevents premature termination of treatment.

Shousha et al. found that UHR-OCT is a good technique when visualizing conjunctival and corneal intraepithelial neoplasia (CCIN). UHR-OCT is particularly useful for the confirmation of recovery of CCIN. This is important because UHROCT could in this way replace a biopsy, which is harmful to 
the surface of the eye and which can be false negative because of sampling error. Disadvantages of UHR-OCT concerning CCIN are the fact that microinvasion cannot be excluded and the fact that the resolution of UHR-OCT is not high enough to assess intracellular characteristics [9].

UBM and AS-OCT both have a strong correlation with histopathology and they can both assess the structure and the extent of lesions in order to guide treatment $[8,10,24]$. When tumor invasion of the sclera or cornea is documented before resection, the surgeon can prepare and counsel the patient for a more extensive resection with graft or even discuss the possibility of an enucleation or exenteration.

A major limitation of most studies was the small study population, often leading to the conclusion that further research is needed. Furthermore, only a limited amount of tumor types was investigated, which makes it impossible to extrapolate these findings to all corneal and conjunctival tumors.

\section{Conclusion}

The literature shows that AS-OCT and UBM are both very useful and complementary techniques for the evaluation and follow-up of corneal and conjunctival tumors even though they cannot replace histopathological analysis for the diagnosis. Due to their different measuring technique, they have different advantages and disadvantages. The disadvantage of AS-OCT is that it cannot penetrate deeper than $1-3 \mathrm{~mm}$ and cannot penetrate through pigmented lesions. But for smaller lesions AS-OCT is a more accurate technique that can give detailed images of the remaining healthy cornea, can identify cysts, or might be useful in detecting tumor recurrence. For larger or pigmented lesions UBM can better delineate tumor margins and tumor thickness. More comparative studies are needed to investigate which imaging technique is most suitable for a certain tumor type.

\section{Competing Interests}

The authors declare that there is no conflict of interests regarding the publication of this paper.

\section{Authors' Contributions}

Katleen Janssens and Michelle Mertens contributed equally to this work.

\section{References}

[1] H.-J. Buchwald, A. Müller, J. Kampmeier, and G. K. Lang, "Optical coherence tomography versus ultrasound biomicroscopy of conjunctival and eyelid lesions," Klinische Monatsblätter für Augenheilkunde, vol. 220, no. 12, pp. 822-829, 2003.

[2] R. De Keizer and L. Razzaq, "Imaging of iris melanotic lesions and corneal tumors with three different high speed optical coherence tomography instruments," Acta Ophthalmologica Scandinavica, vol. 85, no. s240, 2007.

[3] A. F. Fercher, W. Drexler, C. K. Hitzenberger, and T. Lasser, "Optical coherence tomography-principles and applications," Reports on Progress in Physics, vol. 66, no. 2, pp. 239-303, 2003.
[4] M. Schneider, O. Szekeres, H. Kiss, M. Kis, A. Papp, and J. Németh, "Comparison of thickness values in nine macular subfields using time-domain and spectral-domain optical coherence tomography," Orvosi Hetilap, vol. 154, no. 52, pp. 2059-2064, 2013.

[5] J. P. S. Garcia Jr. and R. B. Rosen, "Anterior segment imaging: optical coherence tomography versus ultrasound biomicroscopy," Ophthalmic Surgery Lasers and Imaging, vol. 39, no. 6, pp. 476-484, 2008.

[6] H. Li, V. Jhanji, S. Dorairaj, A. Liu, D. S. C. Lam, and C. K. Leung, "Anterior segment optical coherence tomography and its clinical applications in glaucoma," Journal of Current Glaucoma Practice, vol. 6, no. 2, pp. 68-74, 2012.

[7] S. Salim, "The role of anterior segment optical coherence tomography in glaucoma," Journal of Ophthalmology, vol. 2012, Article ID 476801, 9 pages, 2012.

[8] J. Z. Kieval, C. L. Karp, M. A. Shousha et al., "Ultra-high resolution optical coherence tomography for differentiation of ocular surface squamous neoplasia and pterygia," Ophthalmology, vol. 119, no. 3, pp. 481-486, 2012.

[9] M. A. Shousha, C. L. Karp, V. L. Perez et al., "Diagnosis and management of conjunctival and corneal intraepithelial neoplasia using ultra high-resolution optical coherence tomography," Ophthalmology, vol. 118, no. 8, pp. 1531-1537, 2011.

[10] M. A. Shousha, C. L. Karp, A. P. Canto et al., "Diagnosis of ocular surface lesions using ultra-high-resolution optical coherence tomography," Ophthalmology, vol. 120, no. 5, pp. 883891, 2013

[11] J. G. Fujimoto, C. Pitris, S. A. Boppart, and M. E. Brezinski, "Optical coherence tomography: an emerging technology for biomedical imaging and optical biopsy," Neoplasia, vol. 2, no. 1-2, pp. 9-25, 2000.

[12] J.-R. Fénolland, M. Puech, C. Baudouin, and A. Labbé, "Imaging of the iridocorneal angle in glaucoma," Journal Francais d'Ophtalmologie, vol. 36, no. 4, pp. 378-383, 2013.

[13] H. Ishikawa and J. S. Schuman, "Anterior segment imaging: ultrasound biomicroscopy," Ophthalmology Clinics of North America, vol. 17, no. 1, pp. 7-20, 2004.

[14] S. El-Kady, "Ultrasound biomicroscopy: role in diagnosis of iris and ciliary body tumours," The Medical Journal of Cairo University, vol. 79, no. 2, pp. 81-86, 2011.

[15] D. Bhatt, "Ultrasound biomicroscopy: an introduction," Journal of the Bombay Ophthalmologists' Association, vol. 12, no. 1, 2002.

[16] I. M. Lanzl, J. J. Augsburger, R. W. Hertle, C. Rapuano, Z. Correa-Melling, and C. Santa Cruz, "The role of ultrasound biomicroscopy in surgical planning for limbal dermoids," Cornea, vol. 17, no. 6, pp. 604-606, 1998.

[17] C. A. Grant and D. Azar, "Ultrasound biomicroscopy in the diagnosis and management of limbal dermoid," American Journal of Ophthalmology, vol. 128, no. 3, pp. 365-367, 1999.

[18] J. P. Hoops, K. Ludwig, K.-P. Boergen, and A. Kampik, "Preoperative evaluation of limbal dermoids using high-resolution biomicroscopy," Graefe's Archive for Clinical and Experimental Ophthalmology, vol. 239, no. 6, pp. 459-461, 2001.

[19] H.-J. Buchwald, A. Müller, C. W. Spraul, and G. K. Lang, "Ultrasound biomicroscopy of conjunctival lesions," Klinische Monatsblätter für Augenheilkunde, vol. 220, no. 1-2, pp. 29-34, 2003.

[20] H.-C. Lin, S.-C. Shen, S.-F. Huang, and R. J.-F. Tsai, "Ultrasound biomicroscopy in pigmented conjunctival cystic nevi," Cornea, vol. 23, no. 1, pp. 97-99, 2004. 
[21] V. H. Ho, T. C. Prager, H. Diwan, V. Prieto, and B. Esmaeli, "Ultrasound biomicroscopy for estimation of tumor thickness for conjunctival melanoma," Journal of Clinical Ultrasound, vol. 35, no. 9, pp. 533-537, 2007.

[22] C. L. Shields, I. Belinsky, M. Romanelli-Gobbi et al., "Anterior segment optical coherence tomography of conjunctival nevus," Ophthalmology, vol. 118, no. 5, pp. 915-919, 2011.

[23] M. N. Welch, C. D. Reilly, K. Kalwerisky, A. Johnson, and S. G. Waller, "Pterygia measurements are more accurate with anterior segment optical coherence tomography—a pilot study," Nepalese Journal of Ophthalmology, vol. 3, no. 1, pp. 9-12, 2011.

[24] A. A. Nanji, F. E. Sayyad, A. Galor, S. Dubovy, and C. L. Karp, "High-resolution optical coherence tomography as an adjunctive tool in the diagnosis of corneal and conjunctival pathology," Ocular Surface, vol. 13, no. 3, pp. 226-235, 2015.

[25] C. Bianciotto, C. L. Shields, J. M. Guzman et al., "Assessment of anterior segment tumors with ultrasound biomicroscopy versus anterior segment optical coherence tomography in 200 cases," Ophthalmology, vol. 118, no. 7, pp. 1297-1302, 2011. 


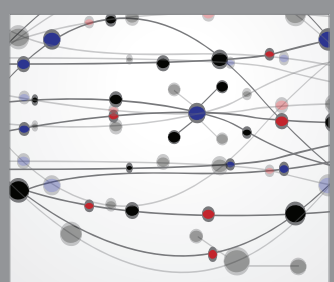

The Scientific World Journal
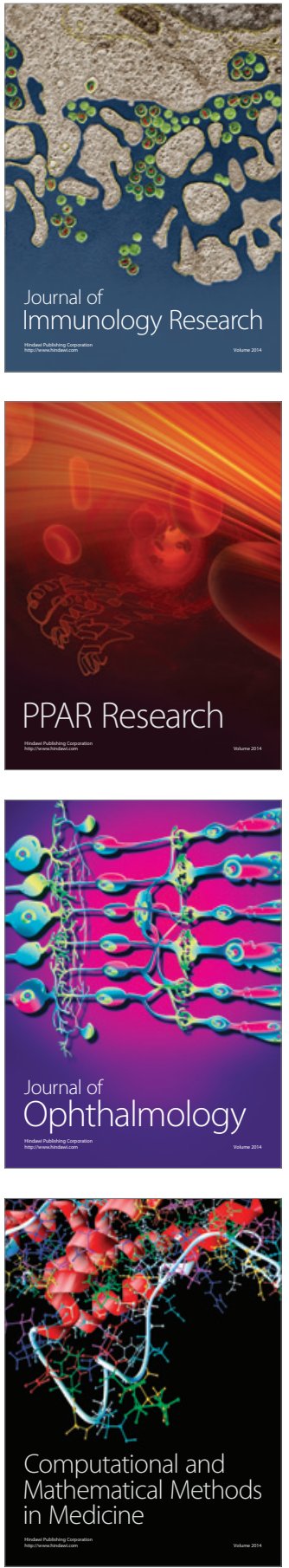

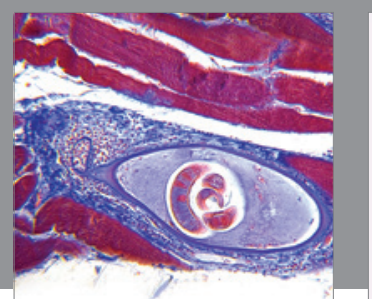

Gastroenterology Research and Practice

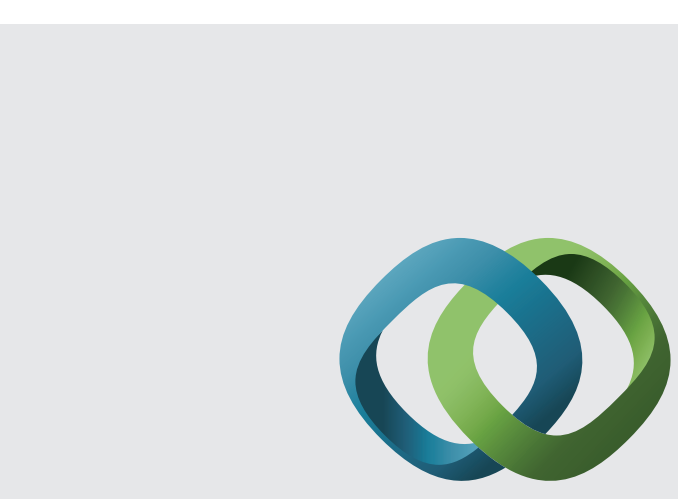

\section{Hindawi}

Submit your manuscripts at

http://www.hindawi.com
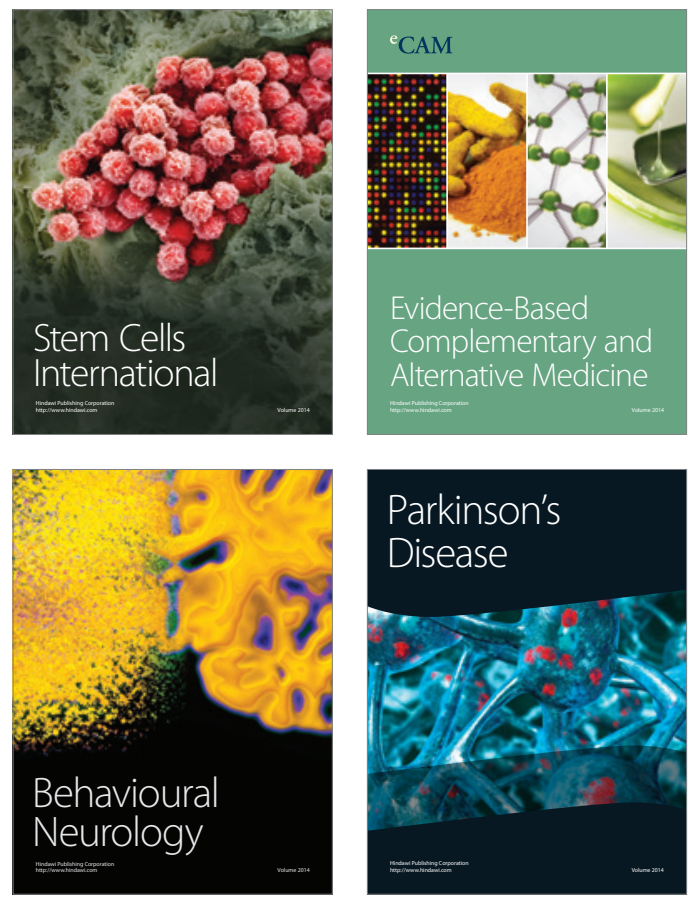
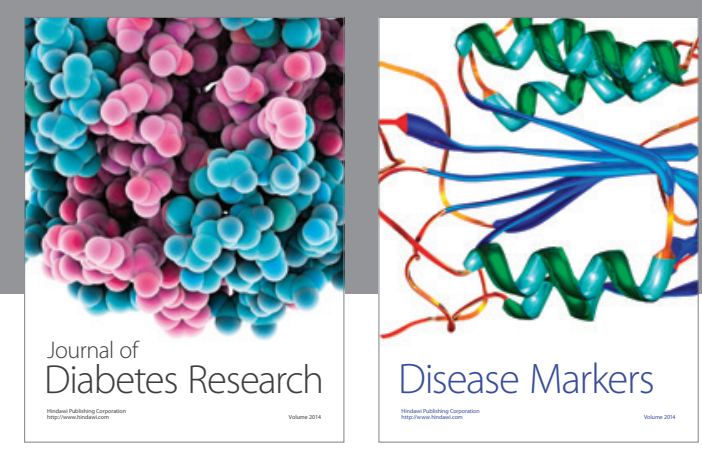

Disease Markers
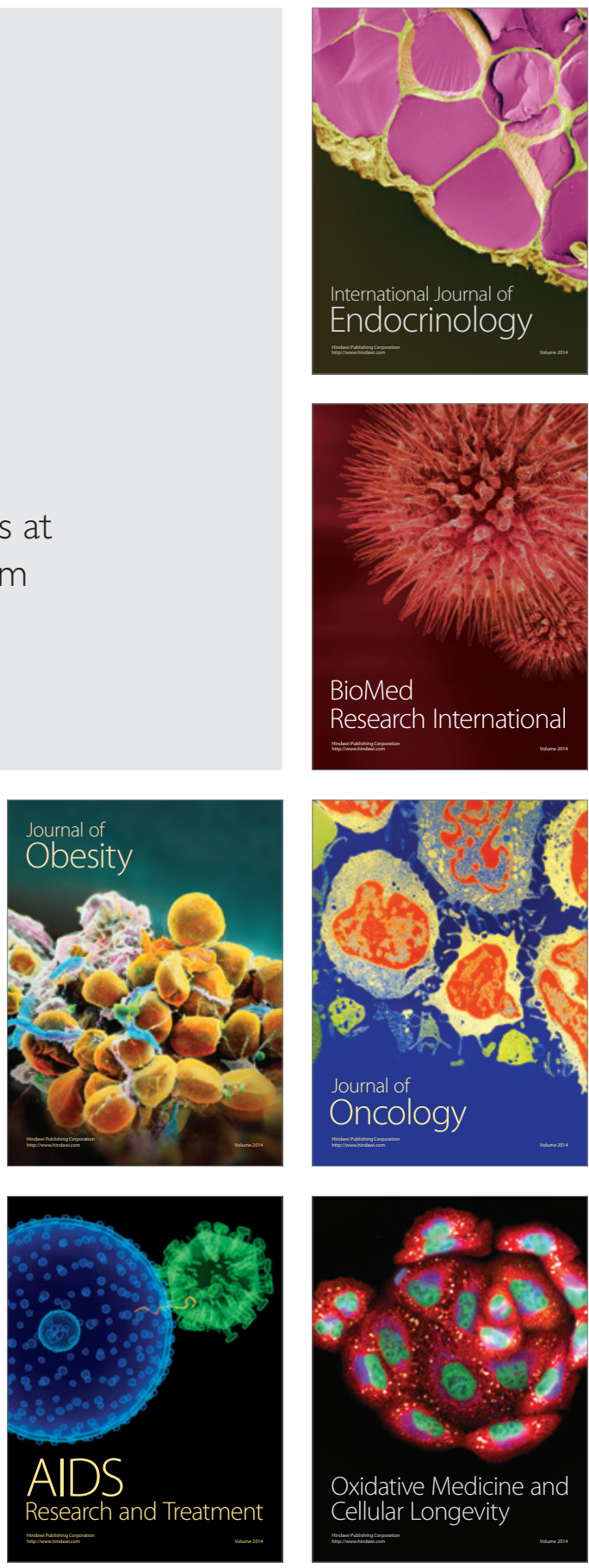